\title{
Duramen y albura de Acacia melanoxylon como materia prima para tableros de partículas
}

\author{
Heart and splint of Acacia melanoxylon as raw material for particleboard \\ PEDRO PELEN, HERNAN POBLETE, LUIS INZUNZA \\ Instituto de Tecnología de Productos Forestales, Universidad Austral de Chile, \\ Casilla 567, Valdivia, Chile.
}

\section{SUMMARY}

The aim of the study is to determine the effect of sapwood and heartwood of Acacia melanoxylon on particleboard properties. Three layer particleboards, $16 \mathrm{~mm}$ in thickness, and $640 \mathrm{~kg} / \mathrm{m}^{3}$ were produced. Boards with 4 sapwood/ heartwood mixtures in proportions of 100/0, 66/33, 33/66, and 0/100 were tested.

Higher content of extractives and lower $\mathrm{pH}$-value in heartwood compared to sapwood were determined.

As the amount of heartwood in the sapwood/heartwood particle mixture increased, the physical-mechanical properties of particleboards improved, except for internal bond (IB). Particleboards with $100 \%$ heartwood presented the highest MOR and MOE values, and the lowest swelling and water absorption values.

Key words: particleboards, heartwood, sapwood, Acacia melanoxylon.

\section{RESUMEN}

El objetivo del estudio es determinar el efecto de la madera de duramen y albura de Acacia melanoxylon sobre las propiedades de tableros de partículas.

Se confeccionaron tableros de tres capas, con $16 \mathrm{~mm}$ de espesor y con una densidad de $640 \mathrm{~kg} / \mathrm{m}^{3}$. Se utilizaron 4 mezclas albura/duramen (\%/\%), en proporciones 100/0, 66/33, 33/66, 0/100.

Se determinó que el duramen presenta un mayor contenido de extraíbles y un menor valor de pH que la albura. A medida que se incrementa la cantidad de duramen se mejoran las propiedades, salvo en el caso de tracción. Los tableros con $100 \%$ de duramen presentaron los mayores valores de MOR y MOE y los menores valores de hinchamiento y absorción de agua.

Palabras claves: tableros de partículas, duramen, albura, Acacia melanoxylon.

\section{INTRODUCCION}

El aromo australiano (Acacia melanoxylon) posee como características un rápido crecimiento y una temprana formación de madera coloreada, duramen. Por su rápido crecimiento, habitualmente se planta en altas densidades, lo que hace necesario ralear, y el producto de estas intervenciones podría ser utilizado como materia prima en la fabricación de tableros de partículas.

El duramen y la albura presentan diferencias anatómicas, estructurales y químicas. Estas diferencias afectan los procesos industriales (Bamber y Fukazawa 1985). Al fabricar tableros de partícu- las con madera de albura y duramen se ha demostrado que existen diferencias en la calidad de los tableros obtenidos (Lelis et al. 1993; Dix y Roffael 1995). En Chile no se han realizado estudios sobre la utilización de ambos tipos de madera en la producción de tableros de partículas.

Entre las propiedades y características de la madera que más afectan la calidad de los tableros se encuentran la densidad y la composición química. La densidad de la madera y la composición química son, a su vez, características que cambian al pasar de madera de albura a duramen.

En el caso de la densidad es indispensable que la densidad del tablero sea superior a la de la 
madera utilizada. Aparte de lo anterior, el cortar, encolar y prensar maderas de diferentes densidades da como resultado tableros de características distintas (Urzúa y Poblete 1980).

La acidez y el contenido de extraíbles de la madera de albura y duramen también son factores importantes de considerar (Poblete et al. 1991). Una variación en la acidez de la madera tiene un efecto directo en la fabricación y en las propiedades de los tableros (Roffael et al. 1975; Lelis et al. 1993; Lelis, 1995). Las reacciones químicas que ocurren durante el fraguado de los adhesivos siguen su curso óptimo con un rango de pH específico para cada tipo de adhesivo y su variación afecta las propiedades de la unión entre las partículas.

La cantidad de extraíbles también puede afectar las propiedades de los tableros. Los extraíbles, sustancias accesorias que son diferentes en albura y duramen, determinan las características químicas de la madera y por lo tanto afectan la producción de los tableros. Ejemplos claros del efecto de estos extraíbles se encuentran en los trabajos de Roffael et al. (1975), Lelis et al. (1993), Pinto y Poblete (1992).

Lelis et al (1993) y Dix y Roffael (1995) determinan que la resistencia a la flexión, en tableros de albura o de duramen, está influenciada por el diferente contenido de extraíbles que poseen ambos tipos de madera. Las propiedades físicas se ven también afectadas por los extraíbles de la madera (Roffael et al. 1975). En este sentido, es importante el tipo y la cantidad de extraíbles, por lo que en especies de duramen diferenciado el tipo de madera (duramen o albura) tiene efecto sobre estas propiedades.

Por lo señalado anteriormente y considerando que la madera de aromo presenta una formación temprana de duramen coloreado, resulta importante investigar respecto a su comportamiento en la fabricación de tableros.

\section{MATERIAL}

Madera. Como materia prima para la elaboración de los tableros de partículas se utilizó madera de duramen y de albura de aromo australiano. Los árboles necesarios para llevar a cabo el estudio tenían 15 años y fueron extraídos desde el predio Los Pinos, perteneciente a la Universidad Austral de Chile, en la ciudad de Valdivia.
Adhesivo. Se utilizó como adhesivo una formulación comercial de ureaformaldehído (Adelite 3087 de la firma Oxiquim), con una concentración de sólidos del 50\%. Al adhesivo se agregó un catalizador para acelerar el proceso de fraguado $\left(\mathrm{NH}_{4} \mathrm{Cl}, 1 \%\right.$ base resina seca). No se incluyeron hidrófobos.

\section{METODO}

Preparación de la madera. Para obtener madera de albura y de duramen fue necesario aserrar las trozas separando ambas porciones. Posteriormente, la madera fue astillada y viruteada para producir partículas. Las partículas fueron secadas para obtener contenidos de humedad de $7.5 \%$ en las capas externas y $3.5 \%$ en la capa media.

Fabricación de los tableros. Los tableros se fabricaron con un espesor de $16 \mathrm{~mm}$ y un formato de $60 \mathrm{~cm}$ por arista. Se consideró una densidad de $640 \mathrm{~kg} / \mathrm{m}^{3}$ y una proporción capa externa/capa media de 1/1. El contenido de adhesivo en la capa externa fue de $9.5 \%$ y en la capa media de $6.5 \%$. Durante el prensado se aplicó una presión máxima de $3 \mathrm{~N}$ y una presión media de 1,5 N. La temperatura de prensado fue fijada en $160^{\circ} \mathrm{C}$ por un tiempo total de $6 \mathrm{~min}$.

Se fabricaron cuatro tipos de tableros diferenciados por la composición de la mezcla de albura y duramen, con ocho repeticiones cada uno; $100 \%$ albura-0\% duramen, $66 \%$ albura-33\% duramen, $33 \%$ albura-66\% duramen, $0 \%$ albura-100\% duramen.

Propiedades de la materia prima. Con el fin de controlar el efecto de algunas características de la albura y del duramen sobre las propiedades de los tableros, se determinaron la densidad básica de la madera, el contenido de extraíbles en etanoltolueno, la acidez de la madera (en una solución al $10 \%$ de partículas en agua destilada con agitación durante cuatro horas a $200 \mathrm{rpm}$ ) y la forma y tamaño de las partículas según metodología de Poblete (1979).

Para obtener un promedio representativo de la densidad de la materia prima se obtuvieron rodelas cada un metro a lo largo del fuste. De las rodelas se extrajeron probetas de 25 x $25 \mathrm{~mm}$. dos de albura y dos de duramen. 
La determinación de extraíbles en etanol-tolueno se llevó a cabo de acuerdo con la norma TAPPI 5 OS-73. Las muestras fueron preparadas teniendo en consideración las proporciones de albura y duramen con que se fabricaron los tableros.

La acidez se determinó con pH-metro en una solución al $10 \%$ de partículas de madera en agua destilada, luego de agitar cuatro horas a $200 \mathrm{rpm}$. Las determinaciones se efectuaron en la solución y en el extracto. El valor de $\mathrm{pH}$ fue medido antes y después del secado en las mezclas de albura y duramen.

La determinación de la forma y tamaño de las partículas se realizó de acuerdo a la metodología descrita por Poblete (1979).

Propiedades de los tableros. Los tableros fueron climatizados de acuerdo a DIN 50014 y se determinaron las siguientes propiedades: Densidad (DIN 52361), Hinchamiento (DIN 52364), Absorción de agua (según ASTM 1037 en probetas DIN 52364), Flexión estática (DIN 52362) y Tracción perpendicular (DIN 52365).

\section{PRESENTACION Y DISCUSION DE RESULTADOS}

Propiedades de la materia prima madera. En el cuadro 1 se presentan los resultados de las propiedades controladas en la madera.

\section{CUADRO 1}

Densidad básica, contenido de extraíbles y valor de $\mathrm{pH}$ de las mezclas de madera albura/duramen. Specific gravity, amount of extracts and $\mathrm{pH}-\mathrm{V}$ alue of heart and splint mixtures.

\begin{tabular}{|lcccc|}
\hline \multirow{2}{*}{ Propiedad } & \multicolumn{5}{c|}{ Tipo de mezcla } \\
& \multicolumn{4}{c|}{ (albura/duramen: \%/\%) } \\
\cline { 2 - 5 } & $100 / 0$ & $66 / 33$ & $33 / 66$ & $0 / 100$ \\
\hline $\begin{array}{l}\text { Densidad básica } \\
\left(\mathrm{kg} / \mathrm{m}^{3}\right)\end{array}$ & 554 & 539 & 529 & 524 \\
\hline Solubles en & & & & \\
etanol-tolueno & & & & \\
$(\%$ bms) & 3.549 & 4.545 & 7.222 & 8.516 \\
\hline Valor de pH & & & & \\
Solución presecado & 6.58 & 5.95 & 5.88 & 5.48 \\
Solución postsecado & 4.36 & 4.10 & 3.78 & 3.50 \\
Extracto presecado & 6.40 & 5.89 & 5.83 & 5.39 \\
Extracto postsecado & 4.42 & 4.20 & 3.98 & 3.49 \\
\hline
\end{tabular}

Densidad básica. Las densidades de albura y duramen y de sus mezclas se presentan en el cuadro 1. Las densidades de las mezclas de albura y duramen fueron calculadas a partir de las densidades promedio de cada una y ponderando por su porcentaje de participación.

Las determinaciones de densidad permitieron demostrar que existen diferencias. En el caso de albura se determinó una densidad de $553.8 \mathrm{~kg} / \mathrm{m}^{3}$ y en duramen $524.5 \mathrm{~kg} / \mathrm{m}^{3}$. La densidad promedio fue de $539.2 \mathrm{~kg} / \mathrm{m}^{3}$, lo que, de acuerdo con la literatura, corresponde a una densidad adecuada para la fabricación de tableros de partículas ya que no requiere de una densidad de tablero demasiado alta para lograr una razón de compresión superior a 1.1 (Urzúa y Poblete 1980).

La densidad de la madera de aromo australiano registra valores dentro del rango citado en la literatura para esta especie. Paz y Melo (1987) determinaron un valor de $520 \mathrm{~kg} / \mathrm{m}^{3}$, Rodríguez y Poblete (1989) obtuvieron un valor de densidad de $558 \mathrm{~kg} / \mathrm{m}^{3}$, Chudnoff (1984) reporta densidades de $557 \mathrm{~kg} / \mathrm{m}^{3}$.

La diferencia de densidad entre las distintas mezclas puede tener una influencia sobre las propiedades de los tableros. Un aumento de la densidad provoca una disminución de la resistencia a la flexión, tracción y módulo de elasticidad, y un incremento en la absorción de agua e hinchamiento (Moslemi 1974, Vital et al. 1974).

Extraíbles. La cantidad de extraíbles que posee el duramen es más del doble de la que posee la albura y similar a la determinada por Rodrigues (1974) (7.58\% en alcohol-benceno). Resultados similares presentan Dix y Roffael (1995) con madera de Larix decidua, registrando un mayor contenido de extraíbles (solubles en etanol-cycloexhano) en duramen que en albura, $4.6 \%$ y $1.8 \%$, respectivamente.

La diferencia en el contenido de extraíbles que se presenta entre albura y duramen es importante ya que existen extraíbles con la propiedad de ser hidrófobos y que afectan las propiedades físicas de la madera (Poblete et al. 1991). Lelis et al. (1993) señalan que pueden influir en las propiedades mecánicas de los tableros de partículas. Conclusiones similares presentan Roffael et al. (1975).

En el presente estudio, debido a la diferencia registrada entre albura y duramen, la cantidad de extraíbles aumenta notoriamente y en forma gradual al aumentar la proporción de duramen en las 
mezclas de partículas. El efecto de los extraíbles sobre las propiedades de los tableros debiera ser proporcional al cambio de la cantidad determinado en las mezclas.

Acidez. Los resultados de $\mathrm{pH}$ antes y después del secado de las partículas se presentan en el cuadro 1 y demuestran que la madera de duramen es más ácida que la de albura. El grado de acidez es mayor a medida que aumenta la proporción de duramen en las mezclas. Esta diferencia posee gran relevancia ya que al trabajar con un adhesivo de fraguado ácido (ureaformaldehído), al aumentar la acidez se mejora el fraguado del adhesivo y se obtienen propiedades físico-mecánicas más altas. Los resultados obtenidos coinciden con los registrados por Dix y Roffael (1995), quienes determinaron en madera de Larix decidua de 20, 40 y 102 años de edad un mayor grado de acidez en el duramen.

Los resultados muestran una reducción del valor de $\mathrm{pH}$ de aproximadamente dos puntos, tanto para el extracto como para la solución luego del secado. Estos resultados concuerdan con lo obtenido por Albin (1975). La variación de pH por causa del secado es explicada por Poblete (1983), al señalar que por acción de la temperatura parte de las hemicelulosas, los grupos acetilo, da origen a ácidos como el acético y el fórmico.

Forma y tamaño de partículas. La geometría de las partículas tiene un efecto comprobado sobre las propiedades de los tableros (Poblete 1985). Con un análisis granulométrico se determinaron la participación porcentual y el coeficiente de esbeltez
(C.E.) de cada clase de tamaño. Los resultados de este análisis se presentan en el cuadro 2.

\section{CUADRO 2}

Largo, espesor y coeficiente de esbeltez (C.E.) de las partículas.

Length, thickness and slenderness ratio (CE) of partióles.

\begin{tabular}{|lcccccc|}
\hline & \multicolumn{3}{c}{ Capa media } & \multicolumn{3}{c|}{ Capa externa } \\
\cline { 2 - 7 } Madera & Largo & Espesor & CE. & Largo & Espesor & CE. \\
\hline Albura & 19.0 & 0.35 & 41.1 & 6.9 & 0.30 & 22.9 \\
Duramen & 19.1 & 0.35 & 42.9 & 8.0 & 0.34 & 23.2 \\
\hline
\end{tabular}

La producción de las partículas no contempló un corte diferenciado para capa externa y media, por lo que presentan espesores similares. La geometría de partícula producida con aromo australiano es similar a la registrada con otras latifoliadas que crecen en Chile (Poblete y Peredo 1990). Entre las partículas de albura y de duramen no existen diferencias importantes, lo que permite descartar un efecto de su forma en las propiedades de los tableros.

\section{PROPIEDADES FÍSICO-MECÁNICAS DE LOS TABLEROS}

Absorción de agua. En el cuadro 3 se presentan los estadígrafos básicos para la absorción de agua después de 2 y 24 horas de inmersión. Debe tenerse presente que no existe una exigencia en las normas para esta propiedad.

Para determinar la influencia del tipo de madera, albura y duramen sobre la absorción, se efec-

\section{CUADRO 3}

Estadísticas básicas para absorción de agua (2 y 24 h inmersión).

Basic statistics of water absorption ( 2 and $24 \mathrm{~h}$ soaking).

\begin{tabular}{|c|c|c|c|c|c|c|c|c|}
\hline \multirow{3}{*}{$\begin{array}{c}\text { Albura / duramen } \\
(\% / \%)\end{array}$} & \multicolumn{8}{|c|}{ Absorción de agua (\%) } \\
\hline & \multicolumn{4}{|c|}{2 horas } & \multicolumn{4}{|c|}{24 horas } \\
\hline & Prom. & Máx. & Mín. & $\mathrm{DE}$ & Prom. & Máx. & Mín. & $\mathrm{DE}$ \\
\hline $100 / 0$ & 85.51 & 105.51 & 68.94 & 1.12 & 97.88 & 124.78 & 75.65 & 1.10 \\
\hline $66 / 33$ & 83.65 & 105.14 & 64.30 & 1.14 & 95.07 & 112.25 & 76.90 & 1.10 \\
\hline $33 / 66$ & 81.09 & 108.95 & 62.69 & 1.17 & 91.68 & 114.47 & 75.84 & 1.11 \\
\hline $0 / 100$ & 77.53 & 99.57 & 56.32 & 1.13 & 88.56 & 109.80 & 67.84 & 1.10 \\
\hline
\end{tabular}

Prom.: Promedio; Max.: Máximo; Min.: Mínimo; DE: Desviación estándar. 
tuó un análisis de correlación entre ambas variables. Los resultados mostraron coeficientes de correlación bajos, 0.252 y 0.350 para 2 y 24 h respectivamente, pero demostraron que existe una asociación entre el tipo de mezcla y la propiedad. Los valores del nivel de significancia al 5\% indican que son significativos con un intervalo de confianza de $95 \%$ de probabilidad.

Se puede observar que a mayor cantidad de duramen en la mezcla, menor absorción de agua. El mejor resultado se obtiene en el tipo de tablero 0/100 (albura/duramen: \%/\%). Dix y Roffael (1995) obtienen resultados similares, determinando en duramen absorciones a las 2 y $24 \mathrm{~h}$ de $18.9 \%$ y $49.4 \%$ respectivamente, y en albura de $49.2 \%$ y $67.6 \%$. Los tableros analizados por Dix y Roffael (1995) tenían por adhesivo urea y melamina, por lo que los niveles alcanzados son menores que los del presente estudio.

Los resultados de absorción a las 2 y 24 horas de inmersión en agua pueden ser explicados por el mayor contenido de extraíbles que posee el duramen. Juacida (1991) señala que las especies que presentan un duramen de fácil diferenciación, como aromo, generalmente presentan una menor permeabilidad en el duramen por depositaciones, extraíbles o accesorios en los lúmenes celulares. De igual forma, Poblete et al. (1991) afirman que existen extraíbles con la propiedad de ser hidrófobos que disminuyen la capacidad de absorción de la madera.

La densidad ponderada de las mezclas con que fueron fabricados los tableros de partículas podría tener alguna influencia en los resultados discutidos anteriormente, debido a que la madera de albura presentó una densidad mayor que la madera de duramen. Al respecto, Moslemi (1974) señala que la absorción es mayor al aumentar la densidad de la madera.

Hinchamiento. En el cuadro 4 se presentan los promedios, máximos, mínimos y desviaciones estándar calculadas para la propiedad hinchamiento a las 2 y $24 \mathrm{~h}$ de inmersión en agua.

Un análisis de varianza, efectuado para determinar la influencia del tipo de mezcla (albura/ duramen) sobre el hinchamiento, demostró que para un nivel de significancia de un 5\% existen diferencias significativas entre los hinchamientos de las diferentes mezclas de albura y duramen. También se llevó a cabo un análisis de correlación, el que indicó que existe una baja correlación entre el hinchamiento y el tipo de mezcla. El coeficiente "r" para hinchamiento a $2 \mathrm{~h}$ fue de 0.18 y de 0.26 para $24 \mathrm{~h}$. Pese a lo anterior, esta relación es significativa a un nivel de significancia de un $5 \%$, por lo que existe asociación entre las variables.

Los resultados presentados en el cuadro 4 muestran una tendencia a disminuir el hinchamiento a medida que aumenta la cantidad de duramen en la mezcla de partículas. Estos resultados podrían explicarse por un mayor y diferente contenido de extraíbles en la madera de duramen. Roffael y Stegmann (1983) señalan que los extraíbles pueden reducir el hinchamiento de la madera. Aparte del efecto hidrófobo de los extraíbles, debe tenerse en cuenta que la albura tiene un valor de $\mathrm{pH}$ más alcalino, lo que podría estar provocando un fraguado menos eficiente del adhesivo.

Lelis et al. (1993) y Dix y Roffael (1995), con madera de Pinus sylvestris y Larix decidua, determinaron que los tableros de albura experimentan

\section{CUADRO 4}

Estadísticas básicas de hinchamiento (2 y 24 h inmersión).

Basic statistics of thickness swelling ( 2 and 24 h soaking).

\begin{tabular}{|c|c|c|c|c|c|c|c|c|}
\hline \multirow{3}{*}{$\begin{array}{c}\text { Albura / duramen } \\
(\% / \%)\end{array}$} & \multicolumn{8}{|c|}{ Hinchamiento $(\%)$} \\
\hline & \multicolumn{4}{|c|}{2 horas } & \multicolumn{4}{|c|}{24 horas } \\
\hline & Prom. & Máx. & Mín. & $\mathrm{DE}$ & Prom. & Máx. & Mín. & $\mathrm{DE}$ \\
\hline $100 / 0$ & 18.90 & 25.39 & 14.39 & 1.15 & 20.45 & 28.47 & 15.97 & 1.15 \\
\hline $66 / 33$ & 19.60 & 26.98 & 14.94 & 1.15 & 20.86 & 28.30 & 16.12 & 1.14 \\
\hline $33 / 66$ & 18.15 & 23.81 & 14.19 & 1.14 & 19.11 & 25.87 & 15.43 & 1.12 \\
\hline $0 / 100$ & 17.70 & 27.30 & 13.40 & 1.20 & 18.68 & 27.99 & 14.28 & 1.18 \\
\hline
\end{tabular}

Prom. $=$ promedio. Max. $=$ máximo, $\mathrm{Min}=$ mínimo, $\mathrm{DE}=$ desviación estándar . 
un hinchamiento mayor que los de duramen. Los autores atribuyen este hecho a la diferencia en el contenido de extraíbles y a que en el duramen los extraíbles se depositan en las paredes celulares dificultando la circulación de líquidos entre las células. Es posible que en aromo la formación de duramen incipiente pueda provocar efectos similares a los señalados anteriormente, así lo demuestran los contenidos de extraíbles presentados en el cuadro 1.

Como en el caso de absorción, los resultados de hinchamiento también pueden haber sido afectados por la densidad de la madera que conforma las mezclas. La densidad es mayor en la madera de albura que en la de duramen, y un aumento de la densidad de la materia prima puede provocar un incremento en el hinchamiento de los tableros.

Según la norma DIN 68761, el hinchamiento máximo aceptado después de 2 y 24 horas es de $8 \%$ y $16 \%$, respectivamente, límite no cumplido por ninguno de los tratamientos. La incorporación de un hidrófobo cambiaría esta situación.

\section{PROPIEDADES MECÁNICAS}

Flexión. Se determinaron el módulo de rotura (MOR) y el módulo de elasticidad (MOE). Los estadígrafos básicos se presentan en el cuadro 5 .

Los resultados comprueban que al incrementarse la proporción de duramen en la mezcla se provoca un significativo aumento del módulo de rotura y de elasticidad. El mejor resultado es alcanzado en el tipo de tablero 0/100. Dix y Roffael (1995) también determinaron resistencias mayores en table- ros fabricados con madera de duramen que en aquellos fabricados con albura.

La norma DIN 68761 exige en MOR un valor mínimo de $18 \mathrm{~N} / \mathrm{mm}^{2}$, requerimiento que fue superado por todos los tableros.

Un análisis de varianza para la relación entre tipo de mezcla (duramen/albura) con MOR y MOE determinó, con un nivel de significancia de un $5 \%$, que existen diferencias significativas entre todos los tipos de tableros, con excepción de los tableros 33/66 y 0/100 en el módulo de elasticidad.

El análisis de correlación realizado entre la flexión y el tipo de mezcla mostró coeficientes de correlación bajos pero significativos con un intervalo de confianza de un $95 \%$. El valor de "r" para 1a resistencia (MOR) es de 0.376 y de 0.468 para el módulo de elasticidad (MOE). De acuerdo con las regresiones existe una tendencia, donde un incremento en la proporción de duramen en la mezcla determina un alza en la propiedad.

Una explicación al cambio de resistencia por un aumento de la madera de duramen es el distinto grado de acidez que presentan las mezclas. El valor de $\mathrm{pH}$ de la madera ejerce gran influencia en el fraguado del adhesivo y por lo tanto en la calidad de la unión entre las partículas del tablero. Poblete (1978) señala que las reacciones químicas que ocurren durante el fraguado de ureaformaldehído siguen su curso óptimo con un valor de $\mathrm{pH}$ de 3 a 3.5. De acuerdo a lo expuesto, es posible que el aumento de los módulos de rotura y elasticidad se deban al mayor grado de acidez de duramen, alcanzando la mezcla $0 / 100$ (albural duramen: \%/\%) un valor óptimo de $\mathrm{pH}(3.5)$, asegurando de esta manera un adecuado fraguado y una buena calidad de la unión entre partículas.

\section{CUADRO 5}

Estadísticas básicas para flexión estática (MOR y MOE).

Basic statistics for bending strength (MOR and MOE).

\begin{tabular}{|c|c|c|c|c|c|c|c|c|}
\hline \multirow{3}{*}{$\begin{array}{c}\text { Albura / duramen } \\
(\% / \%)\end{array}$} & \multicolumn{8}{|c|}{ Resistencia a la flexión $\left(\mathrm{N} / \mathrm{mm}^{2}\right)$} \\
\hline & \multicolumn{4}{|c|}{ MOR } & \multicolumn{4}{|c|}{ MOE } \\
\hline & Prom. & Máx. & Mín. & $\mathrm{DE}$ & Prom. & Máx. & Mín. & $\mathrm{DE}$ \\
\hline $100 / 0$ & 20.44 & 29.04 & 13.01 & 3.35 & 2500 & 3477 & 1917 & 266 \\
\hline $66 / 33$ & 22.80 & 29.65 & 16.00 & 3.21 & 2724 & 3445 & 2162 & 246 \\
\hline $33 / 66$ & 23.39 & 30.71 & 14.09 & 3.32 & 2925 & 4421 & 1775 & 451 \\
\hline $0 / 100$ & 24.31 & 33.67 & 17.30 & 3.61 & 2968 & 3996 & 2212 & 343 \\
\hline
\end{tabular}

Prom. $=$ promedio, $\mathrm{Max} .=$ máximo, $\mathrm{Mm}=$ mínimo, $\mathrm{DE}=$ desviación estándar . 
La densidad de las mezclas utilizadas en la fabricación de los tableros (mayor en la madera de albura) es otro factor que puede haber influido en los resultados. Moslemi (1974) señala que, en términos generales, materias primas de bajas densidades dan propiedades de resistencia superiores a las materias primas de alta densidad.

Tracción. Los valores de resistencia para esta propiedad se presentan en el cuadro 6 .

\section{CUADRO 6}

Estadísticas básicas para tracción.

Basic statistics for internal bond.

\begin{tabular}{|ccccc|}
\hline \multirow{2}{*}{$\begin{array}{l}\text { Albura / } \\
\text { Duramen } \\
(\% / \%)\end{array}$} & \multicolumn{5}{c}{$\begin{array}{c}\text { Resistencia a la tracción } \\
\left(\mathrm{N} / \mathrm{mm}^{2}\right)\end{array}$} \\
\cline { 2 - 5 } & Promedio & Máximo & Mínimo & $\mathrm{DE}$ \\
\hline $100 / 0$ & 0.49 & 0.66 & 0.33 & 0.07 \\
$66 / 33$ & 0.52 & 0.65 & 0.34 & 0.07 \\
$33 / 66$ & 0.48 & 0.65 & 0.29 & 0.06 \\
$0 / 100$ & 0.50 & 0.67 & 0.38 & 0.06 \\
\hline
\end{tabular}

Se observa que el aumento de la proporción de duramen no tuvo influencia en los valores de resistencia a la tracción. Un análisis de varianza, con un nivel de significancia de un $5 \%$, indicó que existen tipos de mezclas que no presentan diferencias significativas entre sí. Estas se agrupan de la siguiente manera: tableros $100 / 0$ y 33/66; tableros $100 / 0$ y $0 / 100$, tableros $66 / 33$ y $0 / 100$, tableros $33 / 66$ y $0 / 100$.

Aparte de lo anterior, un análisis de correlación determinó que no existe un grado de asociación entre la resistencia a la tracción y el tipo de mezcla. Esto quedó de manifiesto por el bajo valor del coeficiente de correlación determinado $(" r "=0.014)$ y principalmente por la significancia $(\mathrm{p}=0.821)$ que posee dicho coeficiente. Se demuestra entonces que la resistencia a la tracción tiende a mantenerse constante a medida que aumenta la proporción de albura en la mezcla que forma el tablero.

Los resultados difieren de lo informado por Lelis et al. (1993), quienes obtuvieron diferencias en tracción entre tableros de albura y de duramen de Pinus sylvestris, determinando una mayor resistencia en los fabricados con madera de duramen. De acuerdo con los autores, esta situación se de- bería a la diferencia en el contenido de extraíbles existente entre ambos tipos de madera.

Para tracción la norma DIN 68763 exige un valor mínimo de $0.4 \mathrm{~N} / \mathrm{mm}^{2}$. Esta exigencia se cumplió con todos los tipos de mezclas de albura y duramen.

\section{CONCLUSIONES}

Los resultados obtenidos permiten exponer las siguientes conclusiones del estudio:

- Las muestras de albura y duramen de aromo australiano analizadas presentan diferencias en características tales como densidad (ligeramente menor en duramen), contenido de extraíbles (mayor en duramen) y $\mathrm{pH}$ (menor en duramen).

- El aumento de la cantidad de albura en la proporción albura/duramen que forma los tableros produce una disminución en los valores de los módulos de rotura y elasticidad en flexión. La resistencia a la tracción perpendicular permanece constante a medida que se incrementa la cantidad de albura en la mezcla.

- El incremento de la cantidad de albura en la proporción albura/duramen que forma los tableros aumenta la absorción de agua y el hinchamiento.

- Los cambios de las propiedades son causados por las diferencias en el contenido de extraíbles y de valor de $\mathrm{pH}$ de las maderas de albura y duramen. En menor grado, también influyó la densidad de ambos tipos.

- Las propiedades mecánicas de los tableros de partículas fabricados con madera de albura y duramen de aromo australiano cumplen con las exigencias impuestas por la norma DIN, salvo en el caso del hinchamiento, por lo que se sugiere el uso de hidrófobos.

\section{BIBLIOGRAFIA}

ALBIN. R. 1975. "Determinación del pH en diversas especies de los renovales de la provincia de Valdivia, Bosque 1 (1): 3-5.

BAMBER, R. K., K. FUKAZAWA. 1985. "Sapwood and Heartwood: A Review", Forest Products Abstracts 8 (9):265-278.

CHUDNOFF, M. 1984. Tropical timbers of the world. United States Department of Agriculture, $464 \mathrm{p}$.

DIX, B., E. ROFFAEL. 1995. "Zum Verhalten des Splint- und Kernholzes der Lärche (Larix decidua) bei der Herstellung 
von feuchtebeständigen Spanplatten unter Einsatz verschiedener Bindemittel", Holz als Roh- und Werkstoff 53. S. 357-367.

JUACIDA, R. 1991. Algunos factores que influyen en la permeabilidad de las maderas. U. Austral de Chile, Facultad de Ciencias Forestales. Publicación Docente $N^{\circ} 3,16$ p.

LELIS, R., E. ROFFAEL, G. BECKER. 1993. "Zum Verhalten von Splint- und Kernholz der Kiefer bei Verleimung mit Diisocianat-Klebstoffen (PMDI) und Harnstofformaldehydharzen (UF-Harzen). Teil 2: Die Verleimung mit Harstofformaldehydharzen (UF-Harzen)", Holz-Zentralblatt. 7. S: $120-121$

LELIS, R. 1995. Zur Bedeutung der Kerninhaltsstoffe obligatorisch verkernter Nadelbaumarten bei der Herstellung von feuchtebeständigen und biologisch resistenten Holzspanplatten, am Beispiel der Douglasie (Pseudotsuga menziesii (Mirb.) Franco. Dissertation. Forstwissenschaftlichen Fachbereichs. Georg August Universität Göttingen, 249 S.

MOSLEMI, A. 1974. Particleboard I. Materials. Southern Illinois University Press, $244 \mathrm{p}$.

PAZ, J., R. MELO. 1987. "Nuevas especies en la producción de celulosa". Celulosa y Papel. Vol. 3: pgs: 13-15.

PINTO, A., H. POBLETE. 1992. "Efecto del catalizador en las propiedades de tableros de partículas con madera de Tepa". Ciencia e Investigación Forestal 6 (2). S: 259-279.

POBLETE, H. 1978. Uniones de madera con adhesivos. Univ. Austral de Chile, Facultad de Cs. Forestales, Publicación técnica $\mathrm{N}^{\circ} 1,43 \mathrm{p}$.

POBLETE, H. 1979. "Inclusión de aserrín en tableros de partículas", Bosque 3 (1): 34-46.

POBLETE, H. 1983. Veränderungen in Holzspänen während der Trocknung und des Pressens zu Holzspanplatten. Dissertation. U. Göttingen, 157 pp.
POBLETE, H. 1985. Influencia del tamaño de la partícula sobre las propiedades mecánicas flexión y tracción en tableros de partículas aglomerados con ureaformaldehído. Simposium Pinus radiata Investigación en Chile. Univ. Austral de Chile, Facultad de Cs. Forestales, Valdivia, pp. 241-256.

POBlete, H., M. PEREDO. 1990. Tableros a base de desechos de contrachapados. Univ. Austral de Chile, Facultad de Ciencias Forestales, Informe de Convenio $\mathrm{N}^{\circ} 182,150 \mathrm{p}$.

POBlete, H., S. RODRIGUEZ, M. ZARATE. 1991. Extraíbles de la madera y sus características y efectos sobre la utilización de esta materia prima. Univ. Austral de Chile. Facultad de Cs. Forestales, Publicación Docente No 34, 51 p.

RODRIGUES, J. 1974. "Pastas de celulosa a partir de madera de Acacia melanoxylon, Acacia mollisima y Acacia pycnantha", Anales I.N.I.A. Serie Recursos Naturales 1: 227-252.

RODRIGUEZ, S. H. POBLETE. 1989. Estudio de aptitud para pulpa y papel de las especies madereras Aromo Australiano, Sequoia y renoval de Raulí. Univ. Austral de Chile, Facultad de Cs. Forestales, Serie Técnica, Informe de Convenio $\mathrm{N}^{\circ} 157,25 \mathrm{p}$.

ROFFAEL, E. W. RAUCH, C. Von BISMARK. 1975. "Formaldehydabgabe und Festigkeitsausbildung bei der Verleimung von Eichenspänen mit Harnstof- formaldehydharzen", Holz als Roh- und Werkstoff 33. S. 271-275.

ROFFAEL, E., G. STEGMANN. 1983. "Bedeutung der HolzExtraktstoffe in chemisch-technologischer Hinsicht". ADHÄSION 7: 7-11.

URZUA, J.D., H. POBLETE. 1980. Utilización silvoagropecuaria de los terrenos de Nadi. Factibilidad técnica de la producción de tableros de partículas utilizando especies que crecen en terrenos de Nadi. Univ. Austral de Chile, Facultad de Cs. Forestales, Convenio SERPLAC X región-U. Austral de Chile, 140 p. 